\title{
Magnetic Induction-Based Positioning in Distorted Environments
}

\author{
Orfeas Kypris, Member, IEEE, Traian E. Abrudan, Member, IEEE, and Andrew Markham \\ Department of Computer Science, \\ University of Oxford, \\ Oxford, U.K. OX13QD \\ E-mails: firstname.lastname@cs.ox.ac.uk
}

\begin{abstract}
Ferrous and highly conductive materials distort lowfrequency magnetic fields and can significantly increase magnetoinductive positioning errors. In this work, we use image theory in order to formulate an analytical channel model for the magnetic field of a quasi-static magnetic dipole positioned above a perfectly conducting half-space. The proposed model can be used to compensate for the distorting effects that metallic reinforcement bars (rebars) within the floor impose on the magnetic field of a magneto-inductive transmitter node in an indoor, single-story environment. Good agreement is observed between the analytical solution and numerical solutions obtained from 3-D finite element simulations. Experimental results indicate that the image theory model shows improvement over the free space dipole model in estimating position in the distorted environment, typically reducing positioning errors by $22 \%$ in $90 \%$ of the cases and $26 \%$ in $40 \%$ of the cases. No prior information on the geometry of the metallic distorters was available, making this essentially a "blind" technique.
\end{abstract}

Index Terms-Position measurement, electromagnetic induction, magnetic dipoles, distortion, conductors, magnetic fields

\section{INTRODUCTION}

$\mathbf{I}$ NDOOR positioning technologies are a current subject of intensive research both in academia and industry as they enable higher level services, such as indoor navigation [1], location-based services [2], first responder applications [3] and fall monitoring [4]. Magneto-induction is an attractive modality for positioning applications due to the fact that low-frequency magnetic fields penetrate most objects and do not suffer from multipath, which is not the case for higher frequency signals, such as WiFi. Magneto-inductive systems have previously been demonstrated in various applications, such as underground animal tracking [5], and they work particularly well when the surrounding space in the vicinity of the transmitter (TX) is void of ferrous objects [6] (Fig. 1a). However, when the TX is placed on the floor of a building with metallic rebars embedded within the concrete (Fig. 1b), the vertical $(z)$ component of the generated magnetic field vector is perturbed, which in turn invalidates the free space channel model [7]. This results to an increase in the relative position error, lowering the overall accuracy of the system. To derive a new channel model that takes into account the presence of conducting material in the vicinity of the TX and $\mathrm{RX}$, one can use image theory.

In previous work by Arumugam et al. [8], [9], complex image theory was used to derive a channel model used to monitor the trajectory of an American football over a football field, assuming that the earth is a semi-infinite conducting halfspace. In other work [10] a theoretical framework using image theory was provided for localization using magneto-inductive wireless sensor networks in pipeline environments. Combined with the fact that in the area of magnetic induction-based localization, to the best of our knowledge there is no prior work involving classical image theory, the latter motivated us to conduct numerical simulations and experiments in order to confirm the validity of classical image theory in a distorted environment.

To solve this challenge, we formulate a new channel model using classical image theory [11]-[13], which assumes a homogeneous, solid perfect electric conductor (PEC) occupying the space from elevation $z=0$ to $z=-\infty$. While in reality we are unlikely to operate above a solid metallic sheet, we show that our model holds well when the TX is positioned above a sparse array of metallic rebars. To validate the model, numerical simulations using the COMSOL Multiphysics software package [14] were carried out in 3$\mathrm{D}$, and the real component of the magnetic flux density B was plotted as a function of position above plane $\mathcal{P}$, where $\mathcal{P}=(\hat{\mathbf{x}}, \hat{\mathbf{y}})$ is the horizontal plane of the rebars, lying at elevation $z=0$. The $3-\mathrm{D}$ version of the model was also compared to 3-D simulations of a dipole above a homogeneous, perfectly conducting half-space. Experimental results demonstrate significant improvement of the position estimate in $z$-axis when using the image theory channel model, as opposed to using the free space channel model.

\section{BACKGROUND ON POSITIONING}

A positioning system is a framework for determining the position of an object in space [15]-[17]. Such systems may operate on different environment and length scales (indoor [18]-[20], outdoor [8], [9], [21], underwater [22]) as well as dimensions (1-D, 2-D, 3-D). The type of application and its requirements will dictate how the information is channeled and processed. A good example in the electromagnetic domain are global positioning systems (GPS) [23], [24]; satellites continuously transmit propagating electromagnetic waves in which position and current time is encoded, so that the receiver can calculate its distance from each satellite using time-offlight estimates. In the acoustic domain, time-of-flight can 
(a)

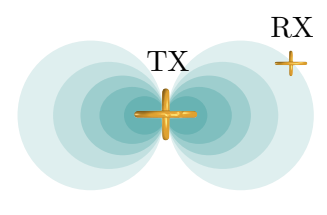

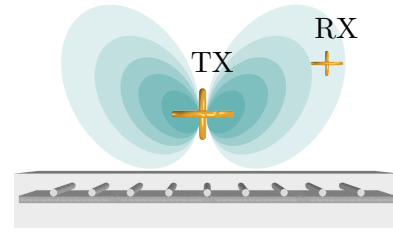

(b)
Fig. 1. Representation of the magnetic field distribution of a magnetoinductive positioning system, depicting a triaxial coil TX and RX a) in free space, and b) over an array of rebars embedded in concrete, which perturb the field symmetry [31].

also be used to calculate relative position of RX to TX by processing the received ultrasonic waves [25]-[27]. Optical systems have also been used for positioning in the past [28].

However, both technologies employ high-frequency propagating waves which are prone to diffraction, refraction, reflection (the cause of multipath propagation, which is when the wave reaches the receiver by two or more paths) and absorption, and thus cannot travel through obstacles (i.e. limited to line-of-sight propagation) [29], [30]. Acoustic systems, even though they can be implemented locally (e.g. within an office space or warehouse) still suffer from the latter effects. Low frequencies, however, penetrate non-conducting objects and attenuate much less with distance, and thus can be used in indoor positioning applications; magnetic-induction based positioning, which is the focus of the present work, makes use of low-frequency magnetic fields.

\section{BACKGROUND ON MAGNETIC INDUCTION-BASED POSITIONING}

Magnetic-induction based positioning systems use coils to generate and detect low-frequency magnetic fields [8], [10], [20], [21], [32]-[37]. In this paper, we use three mutually perpendicular coils, both at transmitter (TX) and receiver (RX), which from now on, will be referred to as triaxial coils. If the physical channel model (i.e. magnetic field distribution) of the system is known, one can obtain position estimates of the RX w.r.t. TX, by optimizing the model against the measured data and extracting the position vector. A position estimate in $\mathbb{R}^{n}$ requires a single TX equipped with $n$ mutually orthogonal coils $n \in\{2,3\}$. Thus, in 2-D one would only need biaxial units, while in $3-\mathrm{D}$, triaxial units are required, provided the transmitter is at an edge of the permitted domain; otherwise an ambiguity in the sign of the position vector $\mathbf{r}$ remains, when both $\pm \mathbf{r}$ are within the allowable domain. In contrast, range based systems require $n+1$ non-collocated transmitters.

\section{A. Magnetic field in free space}

At low enough frequencies, $2 \pi a / \lambda \ll 1$, where $\lambda$ is the wavelength and $a$ is the radius of the coil. This condition makes it possible to treat the TX as an infinitesimal (point) magnetic dipole operating in the near field. In free space, where conducting and/or ferromagnetic objects are absent (Fig. 1a), the magnetic flux density of a point dipole positioned at the origin of the world coordinate frame takes on the following form [7], [38]:

$$
\mathbf{B}(\mathbf{r}, \mathbf{m})=\frac{\mu_{T X}}{4 \pi\|\mathbf{r}\|^{3}}\left(\frac{3 \mathbf{r} \mathbf{r}^{T}}{\|\mathbf{r}\|^{2}}-\mathbf{I}_{3}\right) \mathbf{m}
$$

where $\mathbf{B}(\mathbf{r}, \mathbf{m})$ is the magnetic flux density at the position vector $\mathbf{r}$ due to magnetic dipole moment $\mathbf{m}, \mathbf{I}_{3}$ is the $3 \times 3$ identity matrix, and $\mu_{T X}$ is the permeability of the TX coil, which in our study we consider to be equal to the permeability of free space $\left(4 \pi \times 10^{-7} \mathrm{H} / \mathrm{m}\right)$. Using (1), it is possible to recover the 3-D position of TX w.r.t RX, from measurements of the 3-D magnetic field vector measured at the RX position, in free space [7].

\section{B. Magnetic field in the vicinity of a conducting object}

In the vicinity of conducting objects the free space model in Eq. (1) is no longer valid (Fig. 3). The flux density generated by the TX induces eddy currents within the conductors, which then radiate a secondary (scattered) flux density. Since in our case the eddy-currents can be classified as inductance limited [39], the surfaces of the conductors behave as if they were perfect electric conductors (PEC), and the magnetic field in their vicinity is as if the conductors had zero magnetic permeability (the flux density normal to the surface is close to zero) ${ }^{1}$.

The total flux density $\mathbf{B}^{\mathrm{t}}$ outside the conducting regions will then be equal to

$$
\mathbf{B}^{\mathrm{t}}=\mathbf{B}^{\mathrm{i}}+\mathbf{B}^{\mathrm{s}}
$$

where $\mathbf{B}^{\mathrm{i}}$ is the incident flux density due to the point dipole as in (1), and $\mathbf{B}^{\mathrm{s}}$ is the scattered flux density due to reradiation by the conducting objects. Under the assumption that the objects are perfectly conducting and densely positioned on the plane $z=0$ in a Cartesian co-ordinate system, they can be approximated by an infinitely large electric ground plane that coincides with plane $\mathcal{P}$ (Fig. 2). ${ }^{2}$ At the interface between free space and a PEC, the tangential component of the electric field $\mathbf{E}_{t}$ is continuous, such that the fields on either side of the boundary (field in regions 1 and 2 ) are equal $\left(\mathbf{E}_{t, 1}=\mathbf{E}_{t, 2}\right)$. To solve for the total flux density, there are two alternatives. We can either 1) solve Maxwell's equations in the half-space above the PEC plane taking into account the aforementioned boundary condition, or 2) replace the PEC with free space and place an image dipole at $z=-h$, where $h$ is the distance between the plane of the rebars and the TX (Fig. 2), which will satisfy the aforementioned boundary condition at $z=0$. In the latter case the total flux density $\mathbf{B}^{\mathrm{t}}$ will be a sum of the dipole and image dipole flux densities, such that:

\footnotetext{
${ }^{1}$ If the eddy currents were resistive, the conductors could be perceived as being perfect magnetic conductors (PMC), where the flux density tangential to the surface is close to zero.

${ }^{2}$ The assumption of an infinitely large electric ground plane can only be made when the rebars occur in a grid geometry (parallel sets of rebar crossing), which facilitates the flow of eddy currents and thus the establishment of a scattered field. Solely parallel sets of rebars would impede the flow of eddy currents, much like laminated transformer cores.
} 


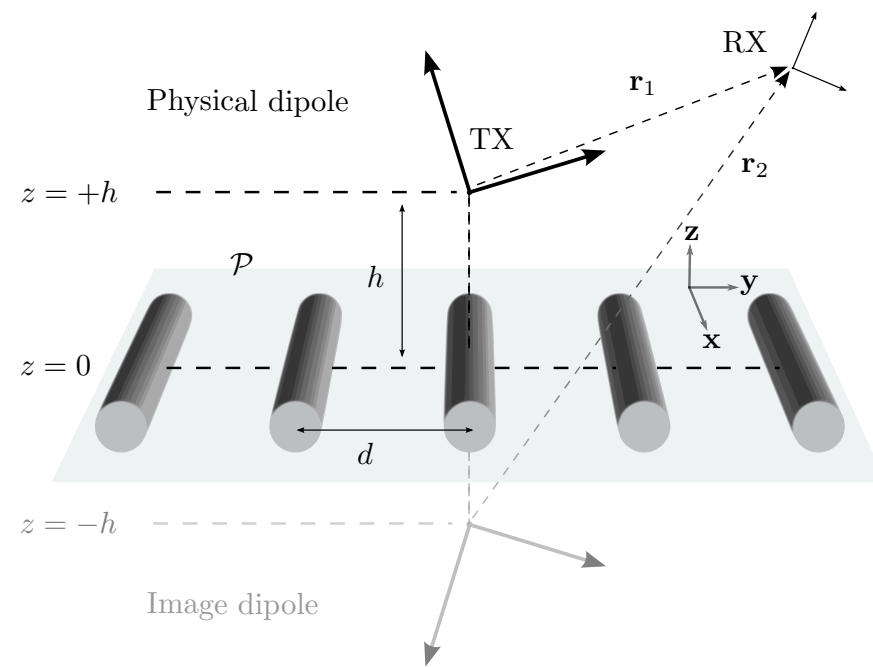

Fig. 2. Illustration of the problem, where the TX is positioned at some height $z=+h$ with its corresponding image positioned at $z=-h$. The position vectors of the RX w.r.t the TX and its image are $\mathbf{r}_{1}$ and $\mathbf{r}_{2}$ respectively. The distance between the geometric centers of the rebars is $d$. The basis coordinate system is denoted as $(\mathbf{x}, \mathbf{y}, \mathbf{z})$.

$$
\begin{aligned}
\mathbf{B}^{\mathbf{t}}(\mathbf{m}) & =\frac{\mu_{T X}}{4 \pi}\left[\left(\frac{1}{\left\|\mathbf{r}_{1}\right\|^{3}}\right)\left(\frac{3 \mathbf{r}_{1} \mathbf{r}_{1}^{T}}{\left\|\mathbf{r}_{1}\right\|^{2}}-\mathbf{I}_{3}\right)\right. \\
& \left.+\left(\frac{1}{\left\|\mathbf{r}_{2}\right\|^{3}}\right)\left(\frac{3 \mathbf{r}_{2} \mathbf{r}_{2}^{T}}{\left\|\mathbf{r}_{2}\right\|^{2}}-\mathbf{I}_{3}\right) \mathbf{M}_{z}\right] \mathbf{m}
\end{aligned}
$$

where $\mathbf{r}_{1}=\left[x_{r}, y_{r}, z_{r}-h\right]^{T}, \mathbf{r}_{2}=\left[x_{r}, y_{r}, z_{r}+h\right]^{T}$ and $x,_{r}, y_{r}, z_{r}$ are the $x, y$ and $z$ coordinates of the RX. $\mathbf{M}_{z}$ denotes a reflection matrix about plane $\mathcal{P}$ (Fig. 2). The above expression is only valid in the region $z>0$, since inside a PEC, $\mathbf{E}=0$, which also implies it cannot support an alternating magnetic field. In the previous expressions it is implicit that TX orientation $\Theta$ and RX orientation $\Omega$ are identical, known, and aligned with the world frame, such that $\boldsymbol{\Theta}=\boldsymbol{\Omega}=\mathbf{I}_{\mathbf{3}}$, where $\boldsymbol{\Omega}$ here is equal to $\left.\boldsymbol{\Omega}\right|_{\langle\mathbb{W}\rangle}$, as seen in the appendix. In the case where $\Theta \neq \Omega$, the definitions seen in the Appendix can be used to relate TX and RX positions and orientations w.r.t. different frames of reference.

\section{Derivation of the channel model}

In previous work [7], an eigenvalue decomposition method was derived in order to obtain the RX position in free space, using vector measurements of the magnetic flux density at the receiver position. The same method, however, cannot be applied to the present analytical expression in Eq. (3), as crossmultiplication terms prevent factorization into separate range and 3-D direction versor. To obtain the magnetic vector field of the TX but in the receiver frame, we need to multiply by $\boldsymbol{\Omega}^{T}$, such that:

$$
\begin{aligned}
\boldsymbol{\Omega}^{T} & {\left[\mathbf{B}^{\mathrm{t}}\left(\mathbf{e}_{\mathbf{1}}\right), \mathbf{B}^{\mathrm{t}}\left(\mathbf{e}_{\mathbf{2}}\right), \mathbf{B}^{\mathrm{t}}\left(\mathbf{e}_{\mathbf{3}}\right)\right]=} \\
& =\frac{\mu_{T X}}{4 \pi} \boldsymbol{\Omega}^{T}\left[\left(\frac{1}{\left\|\mathbf{r}_{1}\right\|^{3}}\right)\left(\frac{3 \mathbf{r}_{1} \mathbf{r}_{1}^{T}}{\left\|\mathbf{r}_{1}\right\|^{2}}-\mathbf{I}_{3}\right)\right. \\
& \left.+\left(\frac{1}{\left\|\mathbf{r}_{2}\right\|^{3}}\right)\left(\frac{3 \mathbf{r}_{2} \mathbf{r}_{2}^{T}}{\left\|\mathbf{r}_{2}\right\|^{2}}-\mathbf{I}_{3}\right) \mathbf{M}_{z}\right] \underbrace{\left[\mathbf{e}_{1}, \mathbf{e}_{2}, \mathbf{e}_{3}\right]}_{\mathbf{I}_{3}}
\end{aligned}
$$

where $\mathbf{e}_{1}, \mathbf{e}_{2}$ and $\mathbf{e}_{3}$ are unit excitation vectors along the $x, y$ and $z$ axes, corresponding to the $\hat{\mathbf{x}}, \hat{\mathbf{y}}$ and $\hat{\mathbf{z}}$-oriented dipoles. We can define the $3 \times 3$ MIMO (multiple-input and multipleoutput) channel matrix: $\mathbf{S}=c \boldsymbol{\Omega}^{T}\left[\mathbf{B}^{\mathrm{t}}\left(\mathbf{e}_{\mathbf{1}}\right), \mathbf{B}^{\mathrm{t}}\left(\mathbf{e}_{\mathbf{2}}\right), \mathbf{B}^{\mathrm{t}}\left(\mathbf{e}_{\mathbf{3}}\right)\right]$, where $c$ is a range-dependent scaling factor $\left(c \propto r^{-2}\right.$ in 2-D and $c \propto r^{-3}$ in 3-D), which also incorporates the TX/RX coils specific parameters (area, number of turns, amplifier gain) and can be determined by calibration from a single measurement taken at a known distance [7]. However, in the case of the image theory model, the scaling factor will differ, so a different procedure common to both models is needed to obtain a calibration; this is described in more detail in Section IV-A.

\section{Simulation}

Numerical solutions for the magnetic field in the presence of steel reinforcement bars were obtained using the AC/DC module within the COMSOL Multiphysics software package. The problem is treated as quasi-static and Ampere's law is used to obtain the magnetic field $\mathbf{H}$ in all regions. In free space, the propagation constant $\gamma=\omega \sqrt{\mu_{0} \epsilon_{0}}$, and in steel, $\gamma=\omega \sqrt{\mu \epsilon}$, where both $\mu$ and $\epsilon$ are complex quantities, and are defined as $\mu=\mu_{0}\left(\mu_{r}^{\prime}-j \mu_{r}^{\prime \prime}\right)$ and $\epsilon=\epsilon_{0}\left(\epsilon_{r}^{\prime}-j \epsilon_{r}^{\prime \prime}\right)$, where $\epsilon_{r}^{\prime \prime}=\sigma / \omega \epsilon_{0}$. To simplify the problem, we assume that the magnetic permeability $\mu$ of steel is real and linear. Ignoring the imaginary part is a reasonable assumption when operating at low frequencies, and assuming a linear permeability is reasonable when operating close to the origin of the $B-H$ curve and below magnetic saturation, which for typical construction steels ranges from 1.0 to $2.0 \mathrm{~T}$. Similarly, it is reasonable to ignore the real part of the complex permittivity, as in the case of conductors $\epsilon_{r}^{\prime \prime} \gg \epsilon_{r}^{\prime}$, owing to their high value of electrical conductivity. For these simulations, $\sigma=1.12 \times 10^{7}$ $\mathrm{S} / \mathrm{m}$ and $\mu_{r}=400$. COMSOL implements the appropriate electromagnetic boundary conditions at the interface between media. To ensure that $\mathbf{H} \rightarrow 0$ as $\mathbf{r}_{\mathbf{1}}, \mathbf{r}_{\mathbf{2}} \rightarrow \infty$, infinite elements were used at the outer boundary of the solution region. To minimize boundary effects even further, the outer boundary was placed at a distance approximately 5 times the width of the region of interest (which was 3 meter radius from the TX).

The magnetic flux density vector calculated by COMSOL is a harmonic quantity, and when the real and imaginary parts are collinear it can be represented as follows:

$$
\mathbf{B}^{\mathrm{t}}=\mathbf{B}_{0}^{\mathrm{t}} e^{j \phi} e^{j \omega t}
$$

where $\mathbf{B}_{0}^{\mathbf{t}}$ only contains spatial information (i.e. magnitude and direction of the vector field), $e^{j \omega t}$ is a time harmonic term which denotes oscillation as a function of time (and can be omitted due to the time periodicity which is implicit), and $e^{j \phi}$ specifies the phase $\phi$ of the field. The quantity that was 


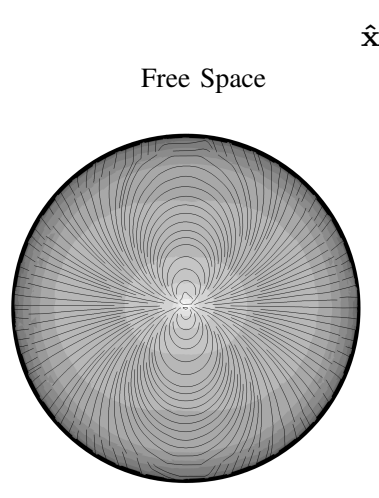

$\hat{\mathbf{x}}$-dipole

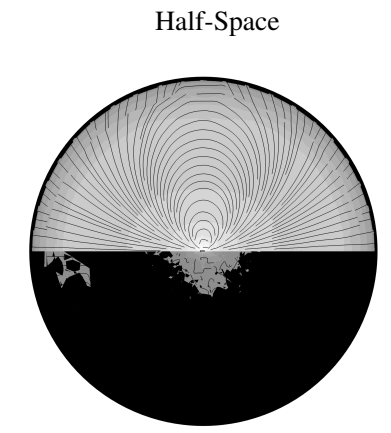

$\hat{\mathbf{z}}$-dipole

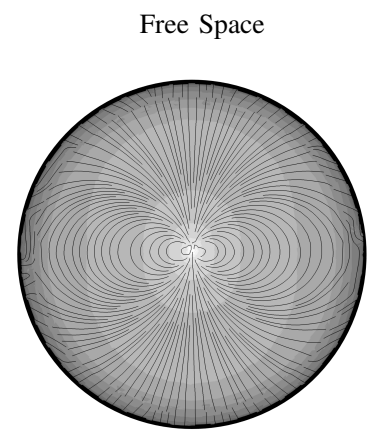

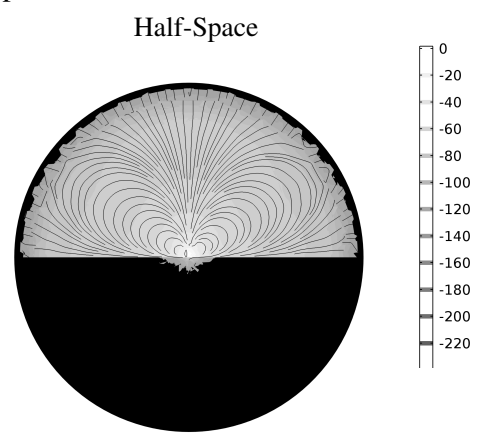

Fig. 3. Flux lines (integral curves) of $\|\mathbf{B}\|$ in $\mathrm{dB}$ obtained from the COMSOL solutions, for the $\hat{\mathbf{x}}$-oriented and $\hat{\mathbf{z}}$-oriented TX dipoles, in the absence of metallic distorters (free-space) and in the presence of a perfectly conducting half-space. Frequency of the magnetic dipoles was set to $2500 \mathrm{~Hz}$. We may notice that the field shape is more distorted for the vertical dipole.

extracted from the COMSOL solution data set is $\mathbf{B}_{0}^{\mathbf{t}}$, which is $\mathbf{B}^{\mathrm{t}}$ evaluated at $\phi=0$, giving the instantaneous value of the field at $t=0$, or equivalently, the real part of the field.

To compare the performance of the free space model, w.r.t. image theory model and numerical model, the angle $\theta$ between vectors corresponding to the two models was calculated, by taking the inner product between data sets, as follows:

$$
\theta=\arccos \left(\frac{\mathbf{B}_{0}^{\mathrm{t}} \cdot \mathbf{B}_{\text {theory }}^{\mathrm{t}}}{\left\|\mathbf{B}_{0}^{\mathrm{t}}\right\|\left\|\mathbf{B}_{\text {theory }}^{\mathrm{t}}\right\|}\right)
$$

The simulations were carried out in 3-D. An infinite halfspace model was used, since the introduction of finite geometries in the form of a 3-D rectangular rebar array would increase the model complexity disproportionately. The mesh was fine-tuned to a maximum element size of $0.1 \mathrm{~m}$ within the region of interest ( $4 \mathrm{~m}$ radius from the TX), in order to avoid discretization errors arising from high field gradients, and was progressively coarsened towards the outer boundary. Again, the real part of the field was extracted from the raw data set.

\section{A. Position Estimation in 3-D}

It is possible to obtain an estimate of parameters $x, y, z$ (free space model) and $x, y, z, h$ (image theory model) by formulating a cost function subject to the following constraints:

$$
\{\hat{x}, \hat{y}, \hat{z}, \hat{h}\}=\underset{x, y, z, h}{\arg \min }\left\|\mathbf{S}_{\text {meas }}-\mathbf{S}_{\text {model }}(x, y, z, h)\right\|_{\mathrm{F}}
$$

where $\|\cdot\|_{F}$ denotes the Frobenius norm, $\mathbf{S}_{\text {meas }}=\left[\mathbf{B}_{\text {meas }}^{\mathrm{t}}\left(\mathbf{e}_{1}\right), \mathbf{B}_{\text {meas }}^{\mathrm{t}}\left(\mathbf{e}_{2}\right), \mathbf{B}_{\text {meas }}^{\mathrm{t}}\left(\mathbf{e}_{\mathbf{3}}\right)\right]$ and $\mathbf{S}_{\text {model }}=c_{\text {model }}\left[\mathbf{B}_{\text {model }}^{\mathrm{t}}\left(\mathbf{e}_{\mathbf{1}}\right), \mathbf{B}_{\text {model }}^{\mathrm{t}}\left(\mathbf{e}_{\mathbf{2}}\right), \mathbf{B}_{\text {model }}^{\mathrm{t}}\left(\mathbf{e}_{\mathbf{3}}\right)\right]$ (since $\boldsymbol{\Omega}=\mathbf{I}_{3}$ ).

In previous work [7] where the model in Eq. (1) was utilized, the position vector was found to be the eigenvector corresponding to the maximal eigenvalue of the Gram matrix $\mathbf{C}=\mathbf{S}^{T} \mathbf{S}$. For this model, it was not yet possible to derive an eigendecomposition method, due to the inseparable terms in the image theory expression (3), thus a grid search optimization algorithm was used to obtain position estimates.
Prior to running the optimization algorithm on the raw data in order to obtain position estimates of RX w.r.t. TX, it was processed in the following way:

1) The measured channel matrices were balanced using experimentally determined coefficients, to account for the slightly different transmit power on each TX axis.

2) A grid search algorithm was written to obtain calibration coefficients, which are used to scale the free space and image theory field amplitudes relative to the measured field. This coefficient includes overall TX and RX gains in the case of the free space model, and in the image theory model it is also a function of $h$.

The resultant $h$ is a nominal distance, and not the actual distance between TX and rebars, as the image theory model assumes an infinite, perfectly conducting half-space and is only an approximation to the sparse array of rebars. We postulate that $h$ is a function of the distance between TX and conductors, amount of conducting material below the TX, the conductivity, as well as the conductor geometry. The aforementioned parameters are not easily decoupled, thus $h$ remains a quantity that must be empirically determined by the above procedure, prior to running the position optimization algorithm. The error estimate in the $x-, y-$ and $z$ directions was calculated by taking the absolute of the difference between estimate and ground truth obtained by the Vicon system.

\section{Results AND ANALYsis}

\section{A. Simulations}

The results of 3-D simulations illustrated in Figs. 4 and 5 provide a more detailed view of the flux density, as one can see clearly how image theory model approximates the field in the presence of a homogeneous half-space, especially for the $\hat{\mathbf{z}}$-oriented dipole.

\section{B. Experiment}

In order to verify the validity of our model, we collected a large number of indoor magneto-inductive measurements in a large hall whose floor contains rebars of unknown geometry and material properties. The nearest walls were 20 meters away, which makes it possible to ignore their influence on 


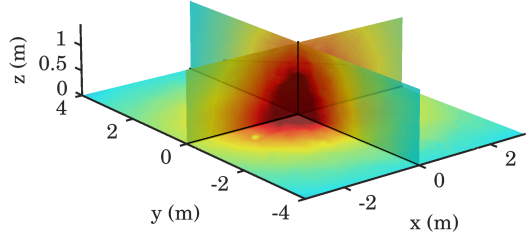

(a)

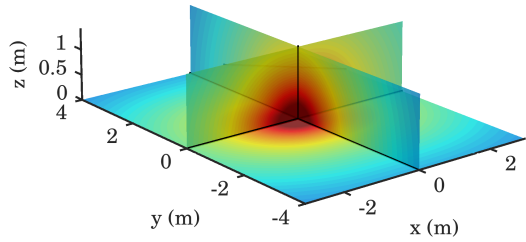

(d)

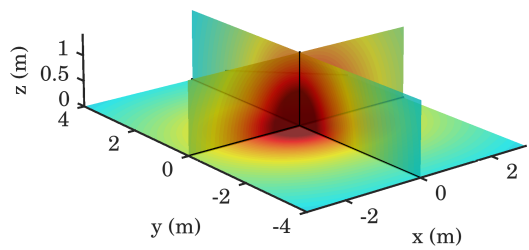

(g)

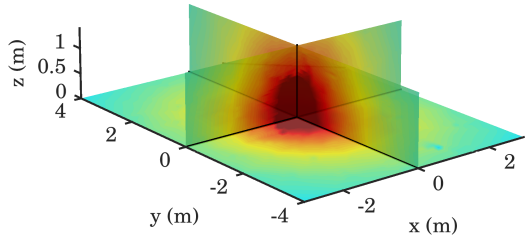

(b)

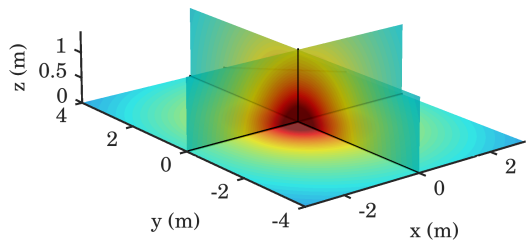

(e)

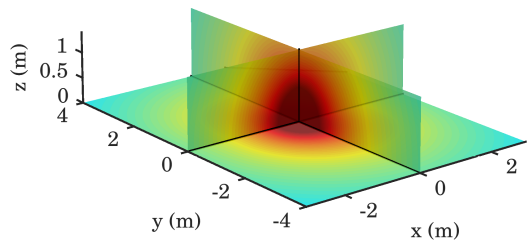

(h)

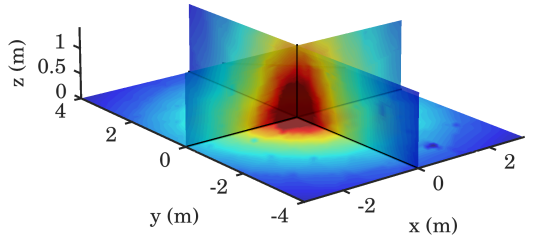

(c)
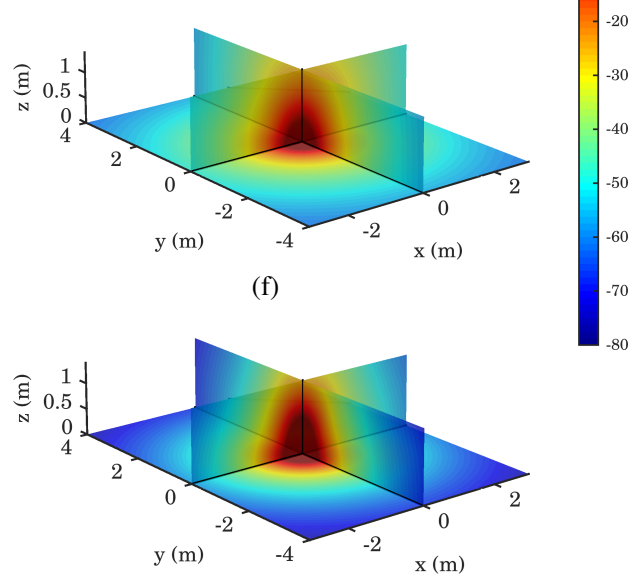

(i)

Fig. 4. $\quad\|\mathbf{B}\|$ for all transmitter axes ( $\hat{\mathbf{x}}, \hat{\mathbf{y}}$ and $\hat{\mathbf{z}}$ oriented dipoles) calculated from the (a)-(c) COMSOL simulation (where the dipole is positioned 0.1 $\mathrm{m}$ above a steel half-space), (d)-(f) free space analytic model, and (g)-(i) image theory model (where an image dipole is used to satisfy the boundary condition $\hat{\mathbf{n}} \times \mathbf{E}=0$ at $z=0$ ). Frequency of the magnetic dipoles was set to $2500 \mathrm{~Hz}$. Best agreement can be seen between COMSOL solution and image theory model, especially for the $\hat{\mathbf{z}}$ oriented dipole. Scale is in $\mathrm{dB}$ below maximum value.

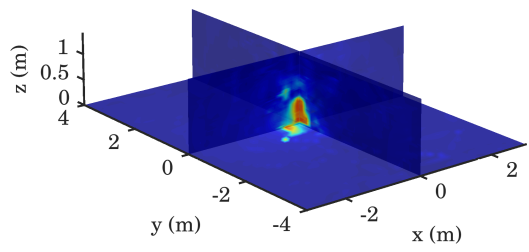

(a)

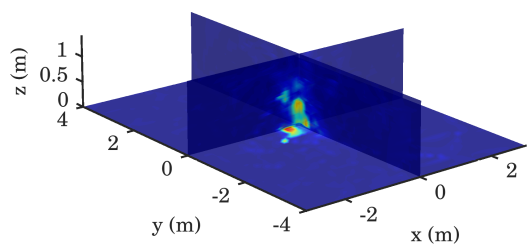

(d)

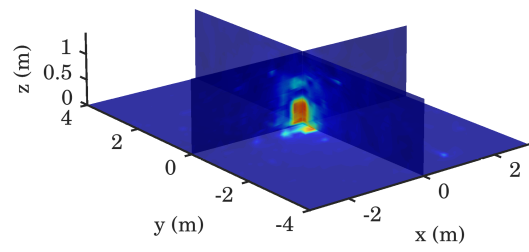

(b)

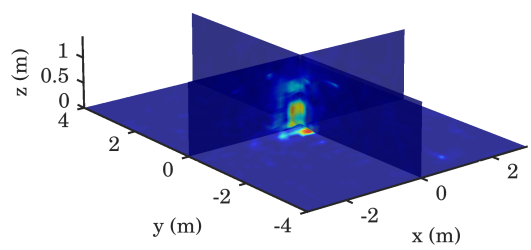

(e)

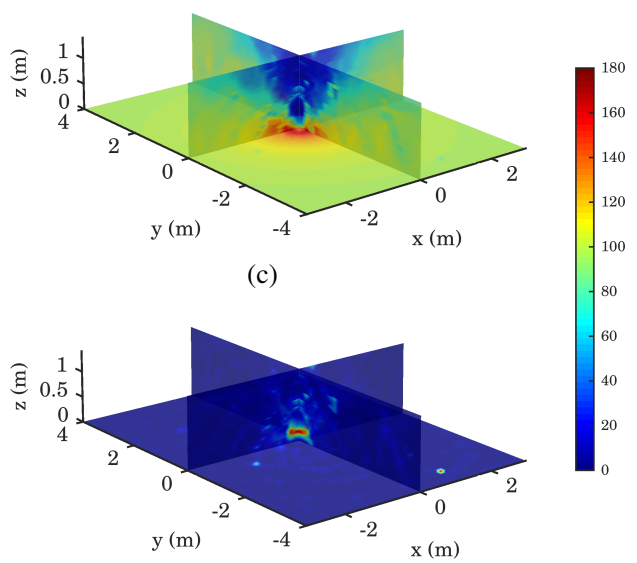

(f)

Fig. 5. Angle of B vector field between COMSOL solution (a)-(c) and free space model, as well as (d)-(f) image theory model, for all transmitter axes ( $\hat{\mathbf{x}}, \hat{\mathbf{y}}$ and $\hat{\mathbf{z}}$ oriented dipoles), calculated from (6). Frequency of the magnetic dipoles was set to $2500 \mathrm{~Hz}$. Best agreement can be seen between COMSOL solution and image theory model, especially for the hatz oriented dipole. The high field gradient in the vicinity of the TX is the cause of numerical error seen in the plots.

the field, even if they were constructed using steel uprights. To the best of our knowledge, except for the rebars in the floor, there was no metal reinforcement structure nearby. The measurement area is shown in Fig. 6. TX was placed on the floor, whereas RX was subsequently placed at 422 different locations enclosed in a volume of $6 \times 8 \times 1.3$ meters. TX and RX orientations were the same as the global coordinate frame, i.e., $\Omega=\Theta=\mathbf{I}_{3}$. Millimeter accuracy ground truth for the RX positions was provided by an optical tracking system consisting of 8 cameras (Vicon T-series with Tracker
2.0 software [40]) placed around the measurement area. To obtain the estimates, the models in Eqs. (1) and (3) were used [7].

By using the aforementioned method prior to running the optimization algorithm, we found that the optimal value for $h$ in our experiment is $0.32 \mathrm{~m}$, which is a sensible nominal separation distance between TX and the plane of the rebars. It is notable that no prior information on the geometry of the metallic distorters was available, and that this is essentially a "blind" technique.

Overall, an improvement in the $z$ position estimate can 


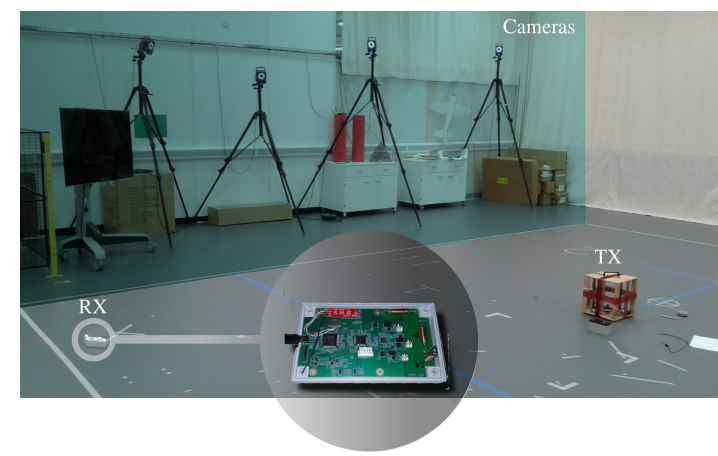

Fig. 6. Measurement area, depicting the TX and RX, as well as the system of cameras used to obtain ground truth [31].

be seen when using the image theory formulation (Figs. 7), while the $x$ and $y$ estimates remain approximately the same. The error estimate improves as the $\mathrm{RX}$ is placed at higher $z$ positions. This can be explained by the fact that the close to the array of rebars the scattered flux density preserves the finer features of the scatterer, while at larger distances from the scatterer these average out, thus approximating a continuous volume of metal.

From Fig. 9, which depicts the spatial error estimates when $\mathrm{RX}$ is positioned at $z=h-0.1 \mathrm{~m}$, a rectangular pattern is discernible in the $z$ estimate. This pattern may be due to the spatial arrangement of the rebars, which on average is less discernible when using the image theory model, as it provides a better approximation to the field in the presence of rebars.

\section{CONCLUSION}

In this work, we derived and experimentally verified a new magneto-inductive channel model that reduces positioning errors in a metal-rich indoor environment. Compared to the free-space model, our model reduces positioning errors by $22 \%$ in $90 \%$ of the cases and $26 \%$ in $40 \%$ of the cases (Fig. 8). This enables applications which require sub-meter positioning accuracy, such as drone position estimation and fall detection.

\section{ACKNOWLEDGMENT}

The authors would like to thank EPSRC for funding this research (Grant ref. EP/L00416X/1 Digital Personhood: Being There: Humans and Robots in Public Spaces (HARPS), and Grant ref. EP/M017583/1 Magneto-Inductive Six Degree of Freedom Smart Sensors (MiSixthSense) for Structural and Ground Health Monitoring), and Dr. Paul Bremner from the Bristol Robotics Laboratory for providing the camera-based measurement facilities (Vicon).

\section{APPENDIX}

\section{DEFINITIONS OF REFERENCE FRAMES}

In order to establish a relationship between the position and orientation of TX and RX, the different frames of reference have to be first defined. The world frame, TX frame, and RX frame are designated as $\langle\mathbb{W}\rangle,\langle\mathbb{T}\rangle$ and $\langle\mathbb{R}\rangle$. Then, TX and $\mathrm{RX}$ positions and orientations are related by the following equations:

$$
\begin{array}{r}
\text { TX position }\left.\right|_{\langle\mathbb{W}\rangle}=\mathbf{t} \\
\text { TX orientation }\left.\right|_{\langle\mathbb{W}\rangle}=\left.\boldsymbol{\Theta}\right|_{\langle\mathbb{W}\rangle}
\end{array}
$$

$$
\begin{array}{r}
\mathrm{RX} \text { position }\left.\right|_{\langle\mathbb{T}\rangle}=\mathbf{r}_{\mathbf{c}} \\
\mathrm{RX} \text { orientation }\left.\right|_{\langle\mathbb{T}\rangle}=\left.\Omega\right|_{\langle\mathbb{T}\rangle}
\end{array}
$$

$$
\begin{array}{r}
\mathrm{RX} \text { position }\left.\right|_{\langle\mathbb{W}\rangle}=\left.\mathbf{t}\right|_{\langle\mathbb{W}\rangle}+\left.\boldsymbol{\Theta}\right|_{\langle\mathbb{W}\rangle} \mathbf{r}_{\mathbf{c}} \\
\mathrm{RX} \text { orientation }\left.\right|_{\langle\mathbb{W}\rangle}=\left.\boldsymbol{\Omega}\right|_{\langle\mathbb{W}\rangle}=\left.\left.\boldsymbol{\Theta}\right|_{\langle\mathbb{W}\rangle} \boldsymbol{\Omega}\right|_{\langle\mathbb{T}\rangle}
\end{array}
$$

In summary, $\left.\boldsymbol{\Theta}\right|_{\langle\mathbb{W}\rangle},\left.\boldsymbol{\Omega}\right|_{\langle\mathbb{T}\rangle}$ and $\left.\boldsymbol{\Omega}\right|_{\langle\mathbb{W}\rangle}$ are rotation matrices, where $\left.\Theta\right|_{\langle\mathbb{W}\rangle}$ rotates from TX to world coordinates, $\left.\Omega\right|_{\langle\mathbb{T}\rangle}$ rotates from RX to TX coordinates, and $\left.\Omega\right|_{\langle\mathbb{W}\rangle}$ rotates from RX to world coordinates.

\section{REFERENCES}

[1] R. Mautz, "Overview of current indoor positioning systems," Geodesy and Cartography, 2009. [Online]. Available: http://www.igp.ethz.ch/geometh/people/rmautz/mautz_estonia08.pdf

[2] D. Mohapatra and S. Suma, "Survey of location based wireless services," in Personal Wireless Communications, 2005. ICPWC 2005. 2005 IEEE International Conference on, Jan 2005, pp. 358-362.

[3] J. Rantakokko, J. Rydell, P. Stromback, P. Handel, J. Callmer, D. Tornqvist, F. Gustafsson, M. Jobs, and M. Gruden, "Accurate and reliable soldier and first responder indoor positioning: multisensor systems and cooperative localization," Wireless Communications, IEEE, vol. 18, no. 2, pp. 10-18, April 2011.

[4] T. Shany, S. J. Redmond, M. R. Narayanan, and N. H. Lovell, "Sensorsbased wearable systems for monitoring of human movement and falls," Sensors Journal, IEEE, vol. 12, no. 3, pp. 658-670, 2012.

[5] A. Markham and N. Trigoni, "Underground localization in 3-D using magneto-inductive tracking," IEEE Sensors Journal, no. 6, pp. 18091816, June 2012.

[6] Z. Sun and I. Akyildiz, "Underground wireless communication using magnetic induction," in Communications, 2009. ICC 'O9. IEEE International Conference on, June 2009, pp. 1-5.

[7] T. E. Abrudan, Z. Xiao, A. Markham, and N. Trigoni, "Distortion rejecting magneto-inductive three-dimensional localization (MagLoc)," IEEE Journal on Selected Areas in Communications, vol. 33, no. 11, pp. 2404-2417, Nov. 2015.

[8] D. D. Arumugam, J. D. Griffin, D. D. Stancil, and D. S. Ricketts, "Threedimensional position and orientation measurements using magnetoquasistatic fields and complex image theory," Antennas and Propagation Magazine, IEEE, vol. 56, no. 1, pp. 160-173, 2014.

[9] D. D. Arumugam, "Position and orientation measurements using magnetoquasistatic fields," Ph.D. dissertation, Citeseer, 2011.

[10] X. Tan, Z. Sun, and P. Wang, "On localization for magnetic inductionbased wireless sensor networks in pipeline environments," in Сотmиnications (ICC), 2015 IEEE International Conference on. IEEE, 2015, pp. 2780-2785

[11] J.-M. Jin, Theory and computation of electromagnetic fields. Hoboken and N.J: Wiley, 2010.

[12] P. Bannister, "The quasi-near fields of dipole antennas," IEEE Transactions on Antennas and Propagation, vol. 15, no. 5, pp. 618-626, 1967.

[13] P. Hammond, "Electric and magnetic images," Proceedings of the IEEPart C: Monographs, vol. 107, no. 12, pp. 306-313, 1960.

[14] COMSOL AB, "COMSOL Multiphysics ( ," Stockholm, Sweden. [Online]. Available: http://www.comsol.com

[15] J. Wang, R. K. Ghosh, and S. K. Das, "A survey on sensor localization," Journal of Control Theory and Applications, vol. 8, no. 1, pp. 2-11, 2010.

[16] S. Gezici, Z. Tian, G. B. Giannakis, H. Kobayashi, A. F. Molisch, H. V. Poor, and Z. Sahinoglu, "Localization via ultra-wideband radios: a look at positioning aspects for future sensor networks," Signal Processing Magazine, IEEE, vol. 22, no. 4, pp. 70-84, 2005. 
Error in $\mathrm{x}$
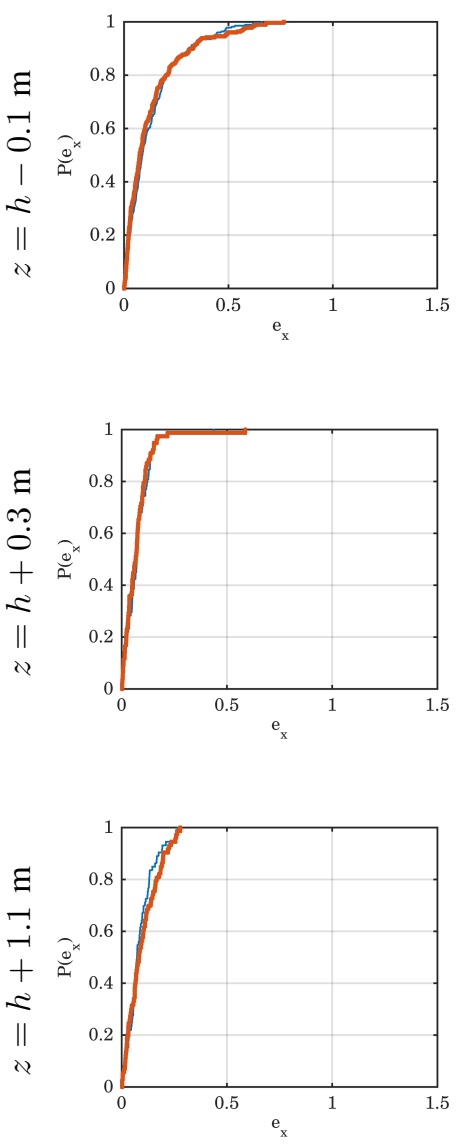

Error in $\mathrm{y}$
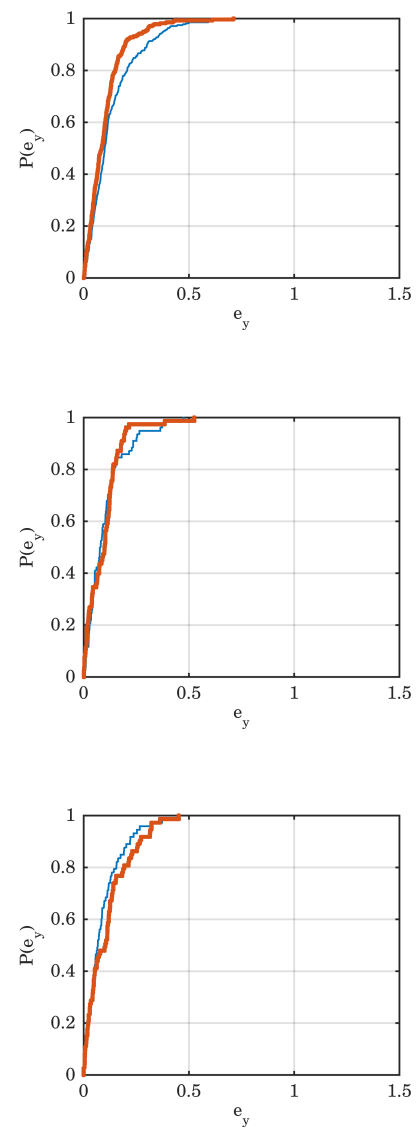

Error in $\mathrm{z}$
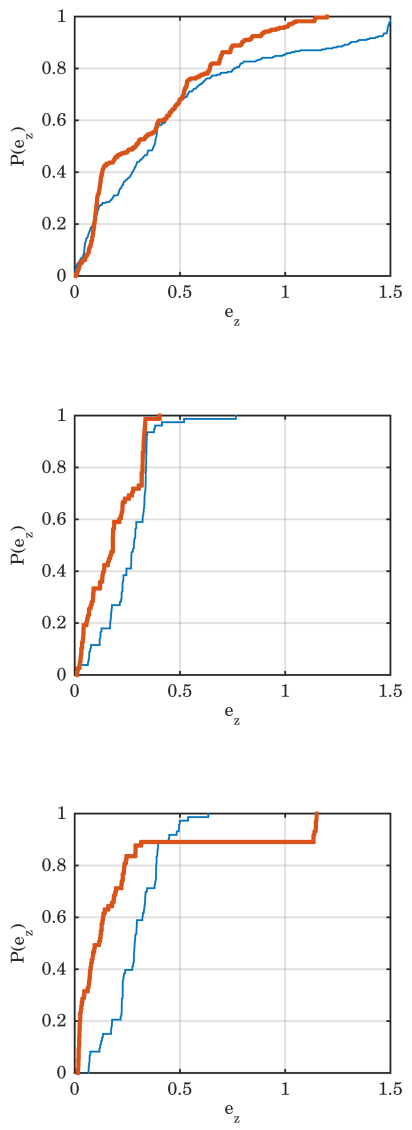

Total Error
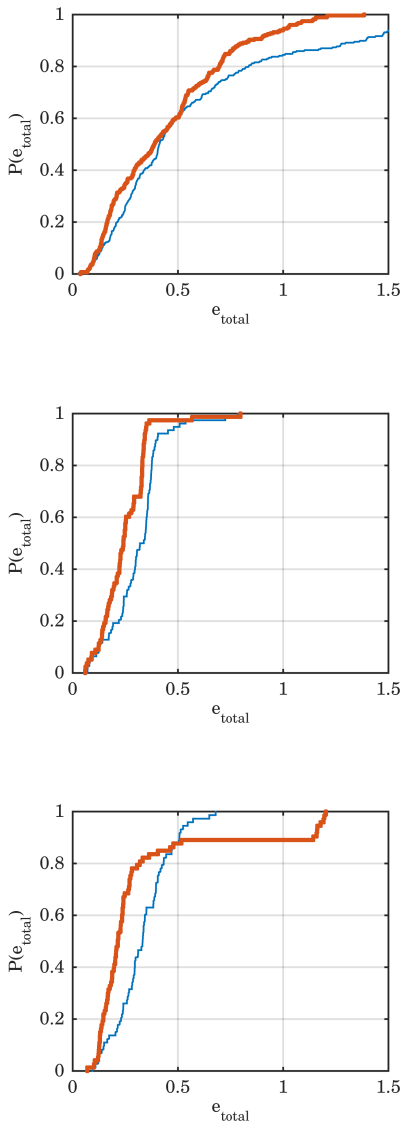

Fig. 7. Cumulative distribution functions $P\left(e_{x}\right), P\left(e_{y}\right), P\left(e_{z}\right)$, of the positioning error estimates $e_{x}, e_{y}, e_{z}$ in $x, y$, and $z$ directions respectively, using the free space model (blue) and image theory model (red), when the Rx is positioned at $z=h-0.1 \mathrm{~m}$ (first row), $z=h+0.3 \mathrm{~m}$ (second row), and $z=h+1.1$ $\mathrm{m}$ (third row).

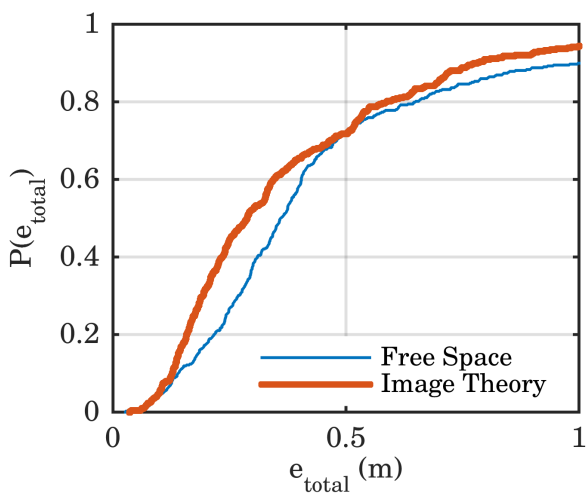

Fig. 8. Cumulative distribution function $P\left(e_{\text {total }}\right)$ of the total positioning error estimates $e_{\text {total }}$, representing the total error for both models. The image theory model is seen to reduce positioning error by $22 \%$ in $90 \%$ of the cases and $26 \%$ in $40 \%$ of the cases.

[17] S. Pandey and P. Agrawal, "A survey on localization techniques for wireless networks," Journal of the Chinese Institute of Engineers, vol. 29, no. 7, pp. 1125-1148, 2006.

[18] J. Rantakokko, J. Rydell, P. Stromback, P. Handel, J. Callmer, D. Tornqvist, F. Gustafsson, M. Jobs, and M. Gruden, "Accurate and reliable soldier and first responder indoor positioning: multisensor systems and cooperative localization," Wireless Communications, IEEE, vol. 18, no. 2, pp. 10-18, 2011.

[19] H. Liu, H. Darabi, P. Banerjee, and J. Liu, "Survey of wireless indoor positioning techniques and systems," Systems, Man, and Cybernetics, Part C: Applications and Reviews, IEEE Transactions on, vol. 37, no. 6 , pp. 1067-1080, 2007.

[20] G. Pirkl and P. Lukowicz, "Robust, low cost indoor positioning using magnetic resonant coupling," in Proceedings of the 2012 ACM Conference on Ubiquitous Computing. ACM, 2012, pp. 431-440.

[21] M. Agrawal and K. Konolige, "Real-time localization in outdoor environments using stereo vision and inexpensive gps," in Pattern Recognition, 2006. ICPR 2006. 18th International Conference on, vol. 3. IEEE, 2006, pp. $1063-1068$.

[22] V. Chandrasekhar, W. K. Seah, Y. S. Choo, and H. V. Ee, "Localization in underwater sensor networks: survey and challenges," in Proceedings of the 1st ACM international workshop on Underwater networks. ACM, 2006, pp. 33-40.

[23] P. Misra and P. Enge, Global Positioning System: Signals, Measurements and Performance Second Edition. Lincoln, MA: Ganga-Jamuna Press, 2006.

[24] B. Hofmann-Wellenhof, H. Lichtenegger, and J. Collins, Global positioning system: theory and practice. Springer Science \& Business Media, 2013.

[25] F. N. Spiess, C. Chadwell, J. A. Hildebrand, L. E. Young, G. H. Purcell, and H. Dragert, "Precise gps/acoustic positioning of seafloor reference points for tectonic studies," Physics of the Earth and Planetary Interiors, vol. 108, no. 2, pp. 101 - 112, 1998. [Online]. Available: http://www.sciencedirect.com/science/article/pii/S0031920198000892

[26] A. Alcocer, P. Oliveira, and A. Pascoal, "Underwater acoustic positioning systems based on buoys with gps," in Proceedings of the Eighth European Conference on Underwater Acoustics, vol. 8, 2006, pp. 1-8.

[27] K. Vickery, "Acoustic positioning systems. new concepts-the future," in 
Free Space

Error in $\mathrm{x}$

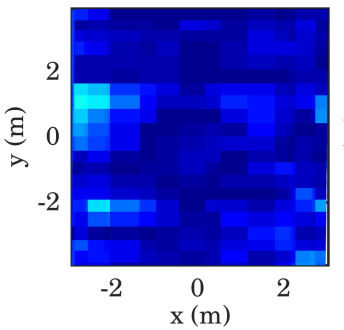

Error in $\mathrm{x}$

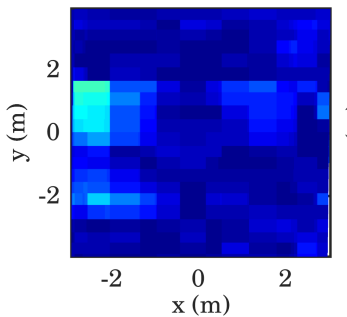

Error in $\mathrm{y}$

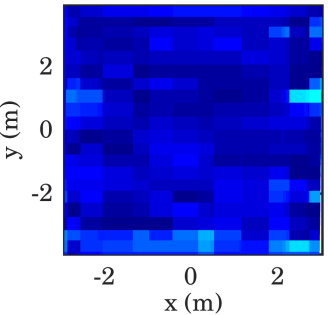

Image Theory

Error in $\mathrm{y}$

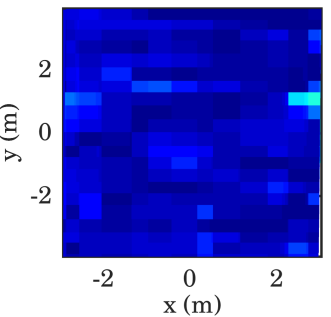

Error in $\mathrm{z}$

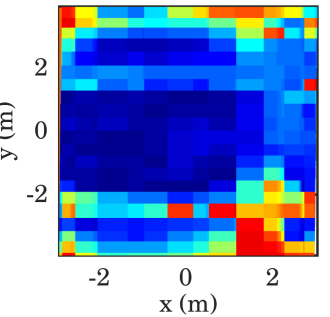

Error in $\mathrm{z}$

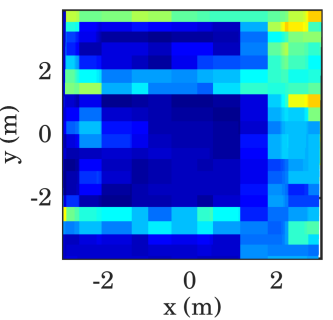

Total Error

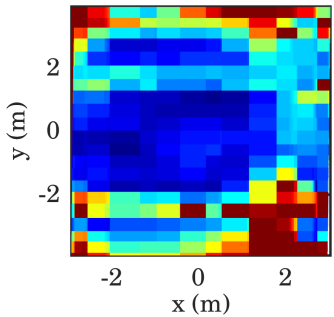

1.6
1.4
1.2
1
0.8
0.6
0.4
0.2
${ }_{0}$

Fig. 9. Spatial errors (Error in $x-, y-$ and $z$ directions) when RX is positioned at $z=h-0.1 \mathrm{~m}$. Overall positioning error is reduced by using the classical image theory model. The systematic error pattern in $z$ may indicate the presence of rebars; the fact that the pattern is linear and not radial supports this assumption.

Autonomous Underwater Vehicles, 1998. AUV'98. Proceedings of the 1998 Workshop on. IEEE, 1998, pp. 103-110.

[28] R. Mautz and S. Tilch, "Survey of optical indoor positioning systems," in Indoor Positioning and Indoor Navigation (IPIN), 2011 International Conference on. IEEE, 2011, pp. 1-7.

[29] G. Dedes and A. G. Dempster, "Indoor gps positioning," in Proceedings of the IEEE Semiannual Vehicular Technology Conference. Citeseer, 2005.

[30] V. Zeimpekis, G. M. Giaglis, and G. Lekakos, "A taxonomy of indoor and outdoor positioning techniques for mobile location services," $A C M$ SIGecom Exchanges, vol. 3, no. 4, pp. 19-27, 2002.

[31] O. Kypris, T. E. Abrudan, and A. Markham, "Reducing magnetoinductive positioning errors in a metal-rich indoor environment," in SENSORS, 2015 IEEE. IEEE, 2015, pp. 1-4.

[32] F. Raab, E. Blood, T. Steiner, and H. Jones, "Magnetic position and orientation tracking system," Aerospace and Electronic Systems, IEEE Transactions on, vol. AES-15, no. 5, Sept. 1979.

[33] J. I. Agbinya, Principles of Inductive Near Field Communications for Internet of Things, ser. River Publishers Series on Communications. River Publishers, 2011.

[34] J. B. Kuipers, Quaternions and Rotation Sequences. A Primer with Applications to Orbits, Aerospace, and Virtual Reality. Princeton University Press, 1999.

[35] A. Markham and N. Trigoni, "Magneto-inductive networked rescue system (MINERS): taking sensor networks underground," in "11th International Conference on Information Processing in Sensor Networks (IPSN 2012), 2012.

[36] A. Markham, N. Trigoni, S. A. Ellwood, and D. W. Macdonald, "Revealing the hidden lives of underground animals using magnetoinductive tracking," in 8th ACM Conference on Embedded Networked Sensor Systems.

[37] A. Sheinker, B. Ginzburg, N. Salomonski, L. Frumkis, and B.-Z. Kaplan, "Localization in 3-d using beacons of low frequency magnetic field," Instrumentation and Measurement, IEEE Transactions on, vol. 62, no. 12, pp. 3194-3201, 2013.

[38] R. P. Feynman, R. B. Leighton, and M. Sands, The Feynman Lectures on Physics. Addison Wesley, 1964, vol. II.

[39] P. Hammond, "The calculation of the magnetic field of rotating machines. part 3: eddy currents induced in a solid slab by a circular current loop," Proceedings of the IEE-Part C: Monographs, vol. 109, no. 16, pp. 508-515, 1962.

[40] Vicon Motion Systems LTD, "Tracker 2.0 囚." [Online]. Available: http://http://www.vicon.com/

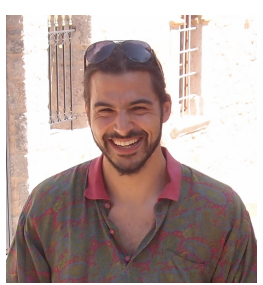

Orfeas Kypris (S'11-M'15) received the B.Eng. degree in Electrical and Electronic Engineering (2009), and the M.Sc. degree in Magnetics (2010) from Cardiff University, Cardiff, U.K. He then joined the Department of Electrical and Computer Engineering at Iowa State University, U.S.A., where he obtained his Ph.D. in Electrical Engineering (2015). Since 2015, he has been a postdoctoral researcher at the Department of Computer Science, University of Oxford, working on indoor localization and structural health monitoring using low-frequency magnetic fields. His research interests include non-destructive evaluation using Barkhausen signals, applied electromagnetism and magnetic materials. He is a member of the IEEE Eta Kappa Nu (IEEE HKN), and the IEEE Magnetics Society.

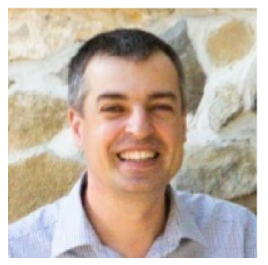

Traian E. Abrudan (S'02-M'09) received the D.Sc. degree (with honors) from Aalto University, Finland (2008), and the M.Sc. degree from the Technical University of Cluj-Napoca, Romania (2000). During 2010-2013, he was a postdoctoral researcher at the Faculty of Engineering, University of Porto, and a member of Instituto de Telecomunicações, Portugal. Since 2013, he has been a postdoctoral researcher at the Department of Computer Science, University of Oxford, working on practical localization algorithms and systems for humans and robots using lowfrequency magnetic fields, as well as other sensing modalities. His fundamental research topics include sensor array signal processing, applied parameter estimation, numerical optimization, and wireless transceiver algorithms. 
Andrew Markham received the Bachelor's (2004) and $\mathrm{PhD}$ (2008) degrees in Electrical Engineering from the University of Cape Town, South Africa. $\mathrm{He}$ is currently an Associate Professor in the Department of Computer Science, at the University of Oxford, working in the Sensor Networks Group. His research interests include low power sensing, embedded systems and magneto-inductive techniques for positioning and communication. 